\title{
Review of studies on SARS-CoV-2 infection inhibitors
}

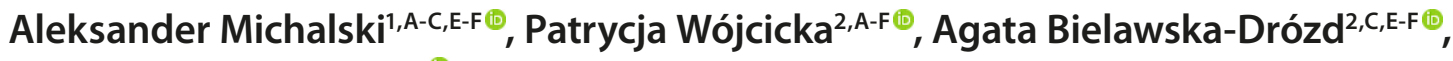 \\ Michał BartoszCze ${ }^{2, A-B, E-F \odot ~}$ \\ 1 1st Clinical Military Hospital with Outpatient Clinic, Lublin, Poland \\ ${ }^{2}$ Biological Threats Identification and Countermeasure Centre, Military Institute of Hygiene and Epidemiology, Warsaw, \\ Poland
}

A - Research concept and design, B - Collection and/or assembly of data, C - Data analysis and interpretation, $D$ - Writing the article, $E$ - Critical revision of the article, $F$ - Final approval of the article

Michalski A, Wójcicka P, Bielawska-Drózd A, Bartoszcze M. Review of studies on SARS-CoV-2 infection inhibitors. Ann Agric Environ Med. 2021; 28(4): 541-550. doi: 10.26444/aaem/143035

\begin{abstract}
Introduction and objective. COVID-19 is a human infectious disease manifested by acute respiratory syndrome. On 30 January, 2020, the Word Heath Organization (WHO) declared a COVID-19 pandemic. The purpose of this article is to review publications on the search for substances that show inhibitory activity against SARS-CoV-2 infectivity, paying particular attention to the effect on different stages of the life cycle of the virus.

Review methods. The review was based on an analysis of the latest available scientific literature and international databases. The data collected relate to the years 2020-2021.

Brief description of the state of knowledge. Extremely intensive research is underway to find compounds that inhibit infection with the SARS-CoV-2 virus. Promising areas of research among the many current endeavours are antiviral compounds that stimulate the immune system, counter proliferation or affect individual viral replication cycles. These include, among others, interferons, monoclonal antibodies, natural compounds, peptides, aptamers, metal salts, and anti-inflammatory agents, inhibitors of viral enzymem, such as the RNA-dependent RNA polymerase. Preparations that help the body to combat the effects of infection have also assumed much importance.

Conclusions. The ongoing research is focused on the development of new antiviral agents, as well as the use of the existing drugs on the market. The results of clinical trials are promising and give hope for the development of effective therapies against SARS-CoV-2 and emerging variants of this virus.
\end{abstract}

- Key words

COVID-19, SARS-CoV-2, antivirals, anti-inflammatories

\section{INTRODUCTION}

COVID-19 is a serious, extremely fast-spreading human infectious disease, the course of which is severe acute respiratory syndrome and the aetiological agent of which is SARS-CoV-2 (severe acute respiratory syndrome coronavirus 2). The disease was first detected in 2019 in Wuhan, Hubei province in China. Despite the imposition of severe restrictions, the disease quickly spread around the world; therefore, on 30 January 30 the World Health Organization (WHO) declared the SARS-CoV-2 COVID-19 pandemic a public health emergency of international concern. As of 10 September 2021, there have been more than 223 million human cases of this disease and more than 3 million deaths worldwide [1].

Structure of SARS-CoV-2. Coronaviruses are members of the Coronaviridae family, which consists of the two subfamilies, Torovirinae and Coronavirinae, the latter of which is divided into four types: alpha, beta, gamma and delta. SARS-CoV-2 is one of the Betacoronaviruses, which is an enveloped RNA virus with a positive-sense, single-stranded genome with a size of approximately $30 \mathrm{~kb}$, encoding 14 open reading

Address for correspondence: Aleksander Michalski, 1st Clinical Military Hospital with Outpatient Clinic, Racławickei 23, Lublin, Poland

E-mail: aleksander.michalski@1wszk.pl

Received: 22.09.2021; accepted: 12.10.2021; first published: 22.10 .2021 frames (ORF). In total, 29 viral proteins have been identified, of which 25 are non-structural and four are structural [2]. The 16 non-structural proteins (NSPs) encoded by ORF1a/ ORF1ab form a replicase-transcriptase complex containing a number of enzymes, such as proteases, polymerases and endonucleases. The four structural proteins are the spike protein $(\mathrm{S})$, a surface fusion protein consisting of two subunits $\mathrm{S} 1$ and $\mathrm{S} 2$; the nucleocapsid protein $(\mathrm{N})$, the membrane protein (M), and the envelope protein (E). The published genome sequences of SARS-CoV-2 indicate existing targets that can be used for COVID-19 treatment [2]. A collection of SARS-CoV-2 samples and sequences has been amassed and is being expanded to monitor emerging strains among the population [3]. Changes in nucleotide sequences diminish the efficacy of monoclonal antibodies used in therapy, strengthen the virus' ability to evade innate or vaccineinduced immunity, and carry the risk that commercial diagnostic tests will work less efficiently [4].

Methods of combating SARS-CoV-2. Three main methods are used to control coronavirus infections: protective vaccination, drug therapy and containment of the spread of the virus. Several viral vaccines are available: vector-based (e.g. AstraZeneca), mRNA (e.g. Moderna, Pfizer/BioNTech) and subunit (e.g. Novavax). Prophylactic vaccination-based coronavirus control strategies have been introduced, mainly targeting the virus $S$ protein, although vaccines targeting 
other molecular targets are being developed aimed at emerging virus variants [5].

The current pandemic caused by SARS-CoV-2 presents many contradictions as to the prevention or treatment methods used [6]. Researchers' efforts are also aimed at finding substances that will inhibit the replication of the virus and the progress of infection. A practical approach is to use preparations already approved and available on the market [2]. Attention has fallen on medicines containing peptides and antibodies or small-molecule chemicals that can interact with enzymes important for viral replication, preparations that help the body fight the effects of infection are also proposed for therapeutic use. Numerous bioinformatics analyses of a number of medicines already on the market have been carried out investigating drug-gene interactions, which have led to the identification of a catalogue of substances with potential to fight SARS-CoV-2 infection. The analyses and clinical trials concern drugs that are already widely used in the treatment of bacterial, fungal, parasitic, and viral infections, as well as anti-inflammatories, antibiotics and natural substances [7].

\section{Life cycle of SARS-CoV-2}

Mechanisms of action of therapeutic agents. In the first stage of infection, the major role in gaining entry into the host cell is played by the $\mathrm{S}$ glycoprotein, which has an affinity for the host cell receptor, the angiotensin-converting enzyme 2 (ACE 2). This enzyme hydrolyses angiotensin II as a cell surface peptidase and is expressed in most organs, particularly the lungs and small intestine [2]. After binding of the viral S protein to the host ACE 2, fusion of the virus membrane with the host occurs, and the virus then enters the host cell by endocytosis. Acidic $\mathrm{pH}$ endosomes are formed where proteolytic cutting of the $S$ protein by transmembrane serine protease 2 (TMPRSS2) occurs [8]. Disruption of the interaction of the $\mathrm{S}$ protein with virus-infected cells can be of significant therapeutic value. In the next stage of infection, viral RNA uses the translation apparatus of the host cell and polyproteins are produced, intersected by $3 \mathrm{C}$-like protease (SARS coronavirus main peptidase) and Papain-like protease produced by ORF1a and ORF1ab, resulting in the formation of all non-structural proteins of the virus. Inhibitors of 3C-like protease and Papain-like proteases have a virusspecific sequence recognising the cut site, and are also a key element for the production of new mature virions [2]. Nonstructural proteins form a replicase-transcriptase complex consisting of the elements necessary for the replication of the viral genetic material and the formation of mRNA, which is the matrix for the translation of the structural S, M, N, and $\mathrm{E}$ proteins of the virus. The proteins produced are then transported to the endoplasmic reticulum of the host cell where mature virions are assembled. These are then released from the cell by exocytosis, after being previously transported in the secretory vesicles to the cell membrane [9]. In the fight against SARS-CoV-2, the strategies being sought are based on the use of drugs that inhibit the growth and multiplication of the virus at each stage of its development [7]. They can inhibit the adsorption of the virus to the host cells, block the fusion of the virus with the membrane of the infected cell, and inhibit the release of nucleic acid from the virus. Inhibitors of viral enzymes find application in this fight, i.e. inhibitors of protease, RNA-dependent RNA polymerase, transcription and cell release [8].

\section{Substances exerting systemic action}

Interferons. Interferons (INFs) are naturally produced glycoproteins in response to pathogens as a part of innate immunity and can be divided into three groups: alpha, beta and gamma. When SARS-CoV-2 enters the host cells, interferon production is inhibited with the level of interferon in the human body therefore correlating with the severity of COVID-19 [10]. Administration of IFN- $\alpha / \beta$ in combination with antiviral agents is a promising therapeutic strategy, and indeed clinical trials have been conducted to evaluate the therapeutic efficacy especially of INF- $\beta$, which was administered intramuscularly in combination with the antiviral agents lopinavir/ritonavir and chloroquine. The trials showed a significant relationship between the use of interferon IFN- $\beta$ in the treatment of COVID-19 and a reduction in the need for intensive care. In addition, a significant decrease in interleukin 6 (IL-6) levels was observed. Interferon therapy reduced hospital stay duration and mortality [11]. Besides interferon, INF- $\alpha 2 b$ also seems to be therapeutically promising. It is a genetically recombinant interferon with additional sugar chains in its structure. Its use also accelerates the elimination of the virus from the respiratory tract and reduces the level of the circulating IL-6 and CRP inflammatory biomarkers. In an interferon-treated group, $95.4 \%$ of patients were discharged from hospital with a negative SARS-CoV-2 test result, while in the control group $26.1 \%$ had the same result. The case fatality rate (CFR) was $0.92 \%$ in interferon-treated patients and $2.95 \%$ in the control group [10]. In vitro studies have revealed that SARS-CoV-2 inhibits the release of INF- $\beta$, which adversely affects the strength of the body's initial innate immune response. It has been observed that in people diagnosed with asthma who are suffering from infections with SARS-CoV-2, those who receive INF- $\beta 1$ recover more quickly. COVID-19 patients were administered nebulised SNG001 (a preparation by Synairgen), which is recombinant INF- $\beta$ for the treatment of upper respiratory tract infections, and were medicated by inhalation to deliver the maximum dose to the lungs. The results of the therapy with SNG001 and statistical analyses indicate that the use of inhaled interferon in COVID-19 patients increases the improvement in the ordinal scale for clinical improvement (OSCI) score two-fold by day 15, and three-fold by day 28, compared to the placebo group. During 14 days of therapy, the probability of recovery was twice as high in the SNG001 group as in the placebo group. In addition, the occurrence of respiratory symptoms, i.e. cough and dyspnoea were milder in interferon-treated subjects than in control group patients [12]. In other studies, a comparative analysis of the effectiveness of interferon administered by aerosol or injection was undertaken. Injectable INF- $\beta$ 1b requires very high doses and has not been effective. Other researchers using nebulised INF- $\alpha 2 b$ showed its positive effect on viral load and the level of interleukin 6 and C-reactive protein biomarkers. Similar results were obtained for inhaled INF- $\kappa$. The administration of interferon in the form of an aerosol allows the dose to be increased and optimised and in this form it reaches the lungs directly, demonstrating a better therapeutic effect. The therapeutic effect of interferons depends on the route and timing of administration and the type of interferon used. The results obtained with interferon therapy are promising and encourage larger-scale clinical trials [12]. 
Acetylsalicylic acid. Acetylsalicylic acid, commonly used in the treatment of cardiovascular diseases, can also be used in the treatment of COVID-19. Studies have shown that COVID-19 duration is shorter and mortality is lower in people taking aspirin. In addition, a lower probability of contracting COVID-19 was observed in a group of patients taking this medication. The host response to and clearance of viral infections depend to a large extent on the expression of type 1 interferon, which modulates lymphocyte response. In addition, in order to evade the immune response of the host, RNA viruses elicit an increase in the level of prostaglandin E2 in the serum, and hence inhibit IFN production and can replicate more quickly [13]. Small doses of aspirin inhibit prostaglandin synthesis, which can enhance the mechanism of the immune response to the virus by inducing IFN 1 secretion. Aspirin directly affects the cGMP-AMP (cyclic guanosine monophosphate-adenosine monophosphate) synthase enzyme, and then the production of signal molecules binding to the STING (stimulator of interferon genes) protein occurs. The STING pathway is activated, which induces the expression of genes encoding immunomodulating molecules, i.e. IFN 1 or cytokines. COVID-19 uses the transmembrane angiotensin-converting enzyme (ACE2) as a transmembrane host cell receptor to infect cells. Higher expression of ACE2 may be beneficial in preventing COVID-19 infection because molecules of the virus may compete with angiotensin-2 protein for cell surface binding sites and uptake by cells. The activation of the STING pathway may be due to overexpression of angiotensin II due to poor conversion of the hormone via ACE2 on the cell surface [13].

Dexamethasone. A glucocorticosteroid with a strong anti-inflammatory and anti-allergic effect. In patients who required mechanical ventilation, responder mortality was observed to be lower at $29.3 \%$ in the dexamethasone-treated group than the $41.4 \%$ rate in the control group. In patients who received standard oxygen therapy and dexamethasone treatment, the mortality rate was $23.0 \%$, and in the placebo group - 26.2\%. Dexamethasone treatment was effective in patients who had symptoms for up to seven days, and increased the likelihood of discharge from hospital within 28 days. Patients receiving oxygen therapy with dexamethasone were less likely to experience clinical deterioration and require mechanical ventilation. No significant effect was observed in patients who were hospitalised but needed no oxygen therapy or invasive ventilation. Dexamethasone is recommended by the European Medicines Agency in COVID-19 cases requiring respiratory support [14].

Colchicine. The main threat to life for COVID-19 patients is an abnormal immune response, not the cytopathic effect caused by virus. Patients with a severe course of virus infection have elevated levels of tumour necrosis factor alpha (TNF- $\alpha$ ), IL-1 and IL-6 inflammatory cytokines and IL-8 chemokine. As a treatment for COVID-19, colchicine inhibits the formation of the nucleotide-binding oligomerisation domain-like receptor family pyrin domain containing 3 (NLRP3) inflammasome, which in turn reduces the release of a range of interleukins [15]. Colchicine is a powerful anti-inflammatory medicine used in the treatment of gout, pericarditis and coronary artery disease, which, when used in the treatment of COVID-19 patients, can be used to treat an excessive immune response in the form of a cytokine storm. The mechanism of action of the drug is to inhibit the polymerisation of tubulin and formation of inflammasomes, cell adhesion molecules and inflammatory chemokines. In the treatment of COVID-19, it reduces the inflammation which causes lung damage and respiratory failure by interfering with the activation and recruitment of leukocytes [15]. Administration of colchicine to patients hospitalised with COVID-19 and with pneumonia confirmed by computed tomography resulted in lower mortality and faster recovery than those of patients in the control group. At 21 days, mortality was $7.5 \%$ in the colchicine group and $28.5 \%$ in the control group. Improvement in health occurred in $40 \%$ of patients treated with colchicine and in $26.6 \%$ of patients in the control group. In $7 \%$ of patients, colchicine treatment was discontinued due to severe adverse reactions, i.e. diarrhoea and skin rash [16].

Allocetra-OTS. These are early apoptotic cells which were originally intended to treat sepsis and have a beneficial effect on cytokine storms. Cell therapy proposed by Israeli researchers [17] involves targeted programming of macrophages, which are key regulators of the immune system. The substance for administration includes mononuclear peripheral blood cells from healthy patients; the therapy consists in transfusing them intravenously to the patient, which stabilises immune system activity and establishes homeostasis. The proposed therapy can be used in a patient at any stage of the disease in whom there are disorders of the immune system. Clinical studies are being conducted on the stability of the proposed therapy. In phase Ib and II clinical trials, $90.5 \%$ of patients who were in critical or serious condition recovered after 28 days, mortality was zero and significant side-effects did not manifest. Further research is needed to determine the exact mechanisms of action of the proposed therapy, its effectiveness and side-effects [18].

Baricitinib. An application has also been submitted for the approval of a product called Olumiant, which contains the active substance baricitinib with an inhibitory effect on inflammation through action on JAK tyrosine kinases. It is a substance used in the treatment of rheumatoid arthritis, which in the treatment of COVID-19 is expected to reduce inflammation and tissue damage in patients requiring oxygen therapy with severe SARS-CoV-2 infection $[19,20]$.

\section{S protein binding inhibition}

Aptamers. A team of researchers have conceived DNA aptamers to fight infection designed to specifically recognise and block the receptor binding domain (RBD) of the $\mathrm{S} 1$ subunit of the viral spike protein [21]. The S1 subunit plays a significant role in the host cell infection process, as it recognises and binds to ACE2, leading to viral infiltration of the host cell and initiation of the SARS-CoV-2 replication process. The aptamers which have been designed have been shown in studies to be effective in inhibiting infection by binding to RBD, thereby limiting the $\mathrm{S} 1$ subunit's ability to recognise and bind to ACE2. The promising results of the proposed solution show it to have high potential for use in antiviral therapy or prophylaxis against SARS-CoV-2 [21]. The many favourable aspects of aptamers make the case for their use. Due to their small molecular size, they can be administered intranasally, and their similarity to nucleic acids ensures their high thermal stability, which facilitates 
transport and storage. In addition, fast kinetics and stability by design are advantageous traits of aptamers. In in vitro studies, shorter aptamer nucleotide sequences with a hairpin structure showed the greatest efficacy in binding to the RBD domain. The shorter sequences demonstrated up to $66.8 \%$ inhibitory effectiveness against infection; by comparison, the control group showed only $1.91 \%$. The results obtained are promising, and ongoing research to design aptamers with different structures and sizes could provide an effective therapeutic solution for the treatment of SARS-CoV-2 infection, and a prophylactic one against such infection [22].

HTCC (N-(2-hydroxypropyl)-3-trimethylammonium chitosan chloride) as polymeric inhibitors based on chitosan. These were the subject of research by a Polish team from the Jagiellonian University in Cracow. They are based on cation-modified chitosan. Studies in vitro and in tissue models have shown that chitosans have very low cytotoxicity and very high efficacy in inhibiting the binding of the $S$ spike protein to the ACE2 cell receptor applicable to almost all coronaviridae viruses. These substances have not yet been authorised for therapeutic use [23].

Artemizan. Artemisia annua extract is routinely used to treat malaria. When administered early in the disease, it shows antiviral activity at the stage of infection in which membrane proteins are involved. In addition, it has anti-inflammatory properties and may reduce the level of inflammatory cytokines and thus protect a patient with mild or moderate symptoms against deterioration of health [24].

Monoclonal antibodies, polyclonal antibodies and cocktails. Monoclonal antibodies are proteins that are designed to attach to a specific structure, which is a viral antigen or important inflammatory receptor. The antibodies considered for SARSCoV-2 attach to its spike protein, which stops the virus from entering the host cells. Preliminary antibody therapy investigations indicate that their use leads to a decrease in the viral load [25]. On the other hand, tocilizumab monoclonal antibody (an interleukin 6 receptor inhibitor which disrupts the cytokine storm) - routinely used for rheumatoid arthritis - has been evaluated in the context of treatment of COVID-19 patients. Patients with hypoxia and other indicators of advanced infection were eligible for the study. After treatment with the proposed antibody, a significant improvement in patients' health was observed [25]. In clinical trials evaluating alternative monoclonal antibody therapy, patients who had tested positive for viral RNA within the 72 hours prior to the study commencement and had experienced their first symptoms within one to seven days previously, were treated with the casirivimab and imdevimab antibody pair active against S protein. Viral RNA levels decreased in patients receiving the antibody therapy compared to those receiving a placebo. The use of the casirivimab and imdevimab monoclonal antibody pair is recommended in the early stages of the disease and in high-risk patients with severe disease. In the later stages of the disease, inflammation and coagulopathy play greater roles than the presence and multiplication of the virus [26]. The health of patients with sustained viral RNA levels in nasopharyngeal swabs over a longer term is more likely to deteriorate. Improvement can be obtained with antibody treatment, which may be an alternative to vaccination in situations immediately after exposure. The effectiveness of this solution is being tested in clinical trials conducted in medical centres [27]. However, multicentre clinical trials do not confirm the effectiveness of plasma from recovered individuals in the standard treatment of COVID-19 patients. There was no significant clinical improvement or reduction in mortality compared to the control group. Patients with a median onset of symptoms of eight days were enrolled [28]. A therapeutic effect in the treatment of COVID-19 using plasma from recovered sufferers was nevertheless observed in the group of patients whose symptoms occurred after three days and to whom the plasma administered had a high antiRBD IgG titre [29]. Combinations of two or more monoclonal antibodies have a higher therapeutic value than monotherapy. In phase three clinical trials, the regdanvimab [CT-P59] monoclonal antibody was effective to the extent of achieving a $70 \%$ reduction in the risk of hospitalisation or death in patients with mild or moderate COVID-19, compared to the placebo group. Studies in vivo in ferrets have shown these antibodies to be effective against newly emerging worldwide variants of SARS-CoV-2, i.e. the B.1.526 (a variant first detected in New York), B.1.525 (a variant detected in Nigeria), B.1.617 (an Indian variant), and B.1.351 (a South African variant) [30].

RB-100. A promising therapeutic agent is the RB-100 monoclonal antibody against the S. protein, which has shown activity in preclinical studies against new variants of SARS-CoV-2 appearing in the UK, South Africa, Brazil, and California and New York in the USA. Its effectiveness against new variants is due to its ability to bind to two different sites of the SARS-CoV-2 spike protein [31].

Sotrovimab. It is a monoclonal antibody for use in highrisk group patients with COVID-19, used in the early phase of infection. Patients with mild to moderate symptoms of infection showed clinical improvement under sotrovimab therapy. The risk of COVID-19 patients' health deteriorating was reduced by $85 \%$ in those receiving sotrovimab treatmen, compared to the placebo group. A monoclonal antibody which neutralises SARS-CoV-2 appears to be an effective response to new variants emerging as a result of changes in the virus genome [32]. The action of the monoclonal antibody targets a conservative epitope which does not include rapidly changing host cell receptor binding motifs, thereby building the strength of the immunological barrier. In studies in vitro, sotrovimab has shown antiviral activity against sarbecoviruses, including variants emerging in the UK, Brazil, South Africa, and California in the USA [32]. The European Medicines Agency is currently analysing data on the use of the casirivimab/imdevimab, bamlanivimab, etesevimab and sotrovimab monoclonal antibodies for the treatment of patients not requiring oxygen therapy, but at the risk of severe disease. Bamlanivimab and etesevimab are monoclonal antibodies targeting the SARS-CoV-2 spike protein. The combination of the two monoclonal antibodies casirivimab and imdevimab (REGN-COV2), targeting the spike protein, is being evaluated, as is sotrovimab [33].

Infliximab. An anti-inflamntory monoclonal antibody used in the treatment of autoimmune disorders, i.e. arthritis and Crohn's disease. Administration to COVID-19 patients is intended to protect against severe infection and worsening clinical status [34]. 


\section{Effect on the ACE2 receptor}

2-deoxy-D-glucose. The virus entering the host cell changes its metabolism so as to create optimal conditions for efficient replication. Disturbances in glucose metabolism accompanying a persistent hyperglycaemic state in people with diabetes may increase the activity of ACE2, and thus increase the infectivity of SARS-CoV-2. The increase in glucose in the body accelerates the replication of the virus and the production of pro-inflammatory cytokines such as TNF- $\alpha$ (tumour necrosis factor alpha) and IL-1 $\beta$. An experiment on a cell line used engineered 2-deoxy-D-glucose which, by inhibiting glycolysis, effectively inhibited the replication of SARS-CoV-2 virus and the expression of ACE2 and proinflammatory cytokines. In diabetics, whose metabolism is disordered by the condition, it is diabetes which explains their increased susceptibility to SARS-CoV-2 infection, and the role of glucose $\mathrm{Z}$ in the induction of pro-inflammatory cytokines increases the risk of severe infection. Glucosetargeted therapy may be an effective antiviral therapy, particularly in individuals with metabolic disorders [35].

Peptides. The research team of Laure et al. [36], have designed a panel of peptides based on the interaction of RBD with the ACE2 receptor. Two peptides were isolated that bound to RBD, inhibiting SARS-CoV-2 infection. Models based on the interaction of RBD with ACE2 allowed the isolation of the six-amino-acid motif of ACE2, which was used to design peptide-based inhibitors. When modelling inhibitors, the following criteria were adopted: interaction with at least three residues interacting with $\mathrm{RBD}$, not being bound in crystalline structures, and being from six to 30 amino acids long. Studies on HEK293T-ACE2 cell lines have demonstrated the ability of three of the six engineered peptides to inhibit viral multiplication. In addition, despite the differences in the genomes of SARS-CoV-2 and SARS-CoV, which are found mainly in the genes encoding the $S$ protein, the binding mechanisms of RBD to the ACE2 receptor were found to be identical and the compositions of the side chains to be conservative. The peptides designed with the EDLFYQ short motif were shown to inhibit SARS-CoV-2 infection, confirming the involvement of the a 1 ACE2 N-terminal helix in the interaction with the virus spike protein. The studies confirm the potential of these peptides in the treatment of COVID-19 [36]. A peptide known as EK1 developed by Xia et al. [37] shows high activity against SARS-CoV-2 as well as MERS and SARS-CoV-1 in animal models. The mechanism of action of the engineered peptide is to inhibit the fusion of the virus with the host cells [37]. Another peptide proposed by a group of researchers led by Zhao [38] is P9, derived from $\beta$-defensin 4. P9 changes the $\mathrm{pH}$ of the endosomes from acidic to alkaline, which prevents the virus from activating the fusion proteins necessary to initiate fusion with the host cells. This mechanism has been shown to be effective not only against SARS-CoV but also against MERS and influenza viruses. In addition, the in vivo experiment by Zhao et al. in a mouse model showed P9 to have therapeutic and prophylactic properties [38].

\section{Effect on virus infiltration of cells}

Budesonide. This synthetic glucocorticosteroid with antiinflammatory and anti-allergic effects can be used in the treatment of COVID-19. Administration of budesonide by inhalation decreases the expression of ACE2 and TMPRSS2, thereby inhibiting viral infiltration of host cells. In in vitro studies using inhaled budesonide, a reduction in SARS-CoV-2 replication in airway epithelial cells resulted. Clinical trials in patients diagnosed with SARS-CoV-2 infection and treated with budesonide showed that this treatment is effective and achieved a three-day reduction in recovery time compared to that of the control group [39].

Camostat mesylate. The preparation is a medicine approved in Japan for the treatment of pancreatitis. It was shown that replication of SARS-CoV-2 can occur not only in the lungs in some patients, but also in the liver, colon, heart, kidneys and blood. Expression of various types of enzyme transmembrane serine, protease 2 (TMPRSS2) was determined. In the liver, colon, heart and blood low-level expression of TMPRSS11D, TMPRSS11E, TMPRSS11F, and TMPRSS13 was observed. TMPRSS2 was expressed at similar levels in kidney, liver and colon cells, although at significantly lower levels than in the lungs. TMPRSS11 and TMPRSS13, expressed in the lungs and blood, were shown to activate virus 37 [40]. The antiviral activity of another serine protease inhibitor has been demonstrated in ex vivo studies. Camostat mesylate is another agent with inhibitive activity with respect to TMPRSS11D and TMPRSS13, which are mainly expressed in the upper respiratory tract. The agent is rapidly hydrolysed in the body to the active metabolite 4-(4-guanidinobenzoyloxy) phenylacetic acid (GBPA) The use of camostat in the treatment of COVID-19 may reduce the risk of multiple organ failure in severe SARS-CoV-2 infection. Anti-inflammatory medicines used to treat pancreatitis, such as camostat and nafamostat, may block the replication of SARS-CoV-2 in lung tissue. SARS-CoV-2 virus enters human cells using the $S$ protein and human ACE2 membrane protein. Then using human enzymes, i.e. TMPRSS2 protease, the $S$ protein is activated, the viral membrane is connected to the target cell and the virus' genetic information is released. The proteases TMPRSS11D and TMPRSS13, which are mainly expressed in the upper respiratory tract, can also be used to activate the $S$ protein. Camostat, its metabolite gamma-aminobutyric acid (GABA) and nafamostat are responsible for blocking the proteases TMPRSS2, TMPRSS13 and TMPRSS11D, which consequently blocks virus entry into cells and its multiplication [40].

Umifenovir. Prevents the entry of the virus into the host cells by inhibiting the fusion of the viral envelope and the cytoplasmic membrane of the host cell and inhibiting endocytosis, in which clathrin participates. It is a medicine used to treat influenza types A and B in Russia and China. Clinical studies showed a decrease in viraemia and mortality in the group of infected patients diagnosed with COVID-19, compared with the control group. It has antiviral activity against hepatitis $\mathrm{C}$, hepatitis $\mathrm{B}$, ebolaviruses, Lassa virus, polio virus and vesicular stomatitis virus [8]. The potential of umifenovir, also known as Arbidol, for COVID-19 treatment is also related to its ability to inhibit the release of SARS-CoV-2 from intracellular vesicles. The effect of the preparation on the two stages of the viral replication cycle can be used both prophylactically and post-exposure [41].

\section{Inhibitors of viral enzymes}

Lopinavir and ritonavir. They are inhibitors of the $3 \mathrm{C}$-like protease 1 viral enzyme which have been used in a combination 
therapy and found to be effective in HIV-infected patients. Lopinavir is an HIV-1 protease inhibitor and is combined with ritonavir, which contributes to an increase in its plasma half-life. In an in vitro study, a combination of these agents was shown to inhibit SARS-CoV, SARS-CoV-2 and MERS. In studies using animal models, there was an improvement in the clinical status of ferrets, but no change in viral load [42]. Clinical trials have been initiated in China, Hong Kong, the Republic of Korea, and Europe. However, clinical trials in patients undergoing COVID-19 treatment with ritonavir in combination with lopinavir did not show a significant difference in patients' viral load or bring improvement in their health [8].

PF-07321332. A serine protease inhibitor that interacts with the cysteine residue of the CL3 viral protease to inhibit the proteolytic cleavage of long viral proteins essential for the viral replication process. In animal model studies, antiviral activity has been demonstrated in vivo. As an agent for oral use, it reaches therapeutic levels in plasma after oral administration [43].

\section{Effect on RNA-dependent RNA polymerase}

Remdesivir (GS-441524). The antiviral activity of this preparation is through the inhibition of the activity of RNA-dependent RNA polymerase. The drug, which is an analogue of adenine nucleotide, is incorporated into viral RNA, disrupting the action of polymerase and inhibiting the elongation of the RNA chain, thereby inhibiting the process of viral replication. It is also a drug originally used to treat Ebola, proposed by the WHO under the 'Solidarity' programme. It acts on viruses that have single-stranded RNA, and studies confirm its effectiveness against SARSCoV2, MERS, and Ebola in preventing the development of infection in respiratory system tissue. Clinical trials of remdesivir have been conducted in patients diagnosed with COVID in a number of countries: the USA, Hong Kong, Republic of Korea, Singapore, Taiwan, France and China. The European Medicines Agency has approved a medicine called Veklury, which contains the active substance remdesivir, for antiviral therapy in the treatment of COVID-19 [8].

Analogue GS-5743. This substance was the subject of research in vitro and animal model studies by an international research team including a group from Poland. Its broadspectrum activity against coronaviruses, including MERS, has been demonstrated by the inhibition of RNA polymerase. Activity against SARS-CoV-2 is also posited [44].

AT-527. An analogue of guanosine and a pro-drug effective in the treatment of liver infections, which has been studied for activity against SARS-CoV-2. When used alone in the treatment of SARS CoV-2, it exhibits strong antiviral activity by selectively inhibiting the enzyme RNA-dependent RNA polymerase. The pharmacologically active form of AT527 arises when it enters the respiratory tract where it is metabolised to AT-9010, which inhibits viral replication. In clinical trials conducted in a group of patients with moderate symptoms of COVID-19, an $80 \%$ decrease in the viral load was noted over two days. As an oral drug without strong side-effects, it has high potential to be a treatment modality for both outpatient and inpatient treatment $[45,46]$.
Favipiravir [T-705]. A pro-drug preparation obtained through chemical synthesis by modification of pyrazine. In Japan, it has been approved for the treatment of influenza. When it enters the body, it is phosphorylated and becomes the active form favipiravir-RTP, which has a structural similarity to guanine, this being a substrate for the enzyme RNAdependent RNA polymerase. Incorporation of the favipiravir molecule by polymerase during replication achieves inhibition of viral protein synthesis. The action of favipiravir at the stage of viral replication provides diverse opportunities for the use of the preparation in antiviral therapy. It also has antiviral activity against influenza viruses, filoviruses, bunyaviruses, arenaviruses, noroviruses, ebolaviruses, and Lassa virus. Clinical studies on favipiravir conducted in several countries, including China, Japan, Saudi Arabia, the USA and India, showed clinical efficacy in the treatment of COVID-19 patients with mild to moderate disease symptoms. In patients with severe COVID-19, favipiravir was not shown to have a beneficial effect on patients' health $[8,47]$.

MK-448/EIDD-2801. This substance is an analogue of the N4-hydroxycytidine (NHC) nucleoside and its mechanism of action is to induce errors in viral replication. It has a broad spectrum of antiviral activity against RNA viruses. Antiviral activity against alphaviruses, pneumoviruses, orthomyxoviruses (influenza viruses) and betacoronaviruses was demonstrated in animal models. In addition to improvement in the health of infected animals, effective suppression of viral spread from infected animals to untreated animals was manifested [48].

Molnupiravir. A pro-drug that acts on the virus after cell infiltration, disrupting the replication process. It is a synthetic analogue of the $\beta$-D-N hydroxycytidine ribonucleoside which is converted into NHC in plasma, and then, by binding to the

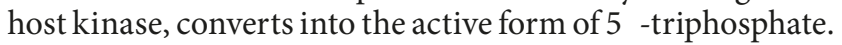
This active form serves as a substrate for RNA-dependent RNA polymerase, causing errors in the newly-formed viral RNA, the number of which increases with each replication cycle. Studies in mice and ferrets treated with molnupiravir showed reduced replication of SARS-CoV-2. Studies in human patients infected with SARS-CoV-2 assessed antiviral activity and the safety of molnupiravir therapy. An RT-PCR study of nasopharyngeal swabs was performed, the virus was isolated on Vero C1008 cells, and samples taken from patients were sequenced. In the samples taken on day three of treatment with molnupiravir (800 $\mathrm{mg}$ ), the prevalence of the infectious virus was only $1.9 \%$ in the treatment group, but was $16.7 \%$ in the placebo group. The viral load in nasopharyngeal swabs also decreased significantly in the treated group. A sequencing study of the mechanism of action of the agent showed twice the frequency of nucleotide changes in RNAdependent RNA polymerase, compared to the placebo group. These results indicate the efficacy of molnupiravir in the treatment of COVID-19. Inhibition of viral replication leads to interruption of development in the early stages of the disease, thereby preventing further transmission of SARSCoV-2 [49].

\section{Immunomodulatory effects}

Factor D Inhibitor. The complement system plays a significant role in the fight against pathogens in the human body and works in concert with the protective functions 
of antibodies. The action of the supplement is exerted by interaction with antibodies, and on an alternative lectin pathway. It is a complex of numerous proteins present in body fluids, the activation of which involves cascading enzymatic and non-enzymatic reactions [50]. Activation of the complement system leads to the production of two enzymes, convertase C3 and convertase C5, and ultimately the pathogen membrane-attacking complex (Mac). It has been observed that coronaviruses have developed the ability to mimic the complement proteins and those of the coagulation system, leading to excessive activation of these systems. In patients with severe COVID-19 who have experienced acute respiratory failure, thrombosis, and embolism, these symptoms validate the role of the complement system in the pathogenesis of COVID-19. Studies in animal models suggest that the increase in respiratory symptoms and the deterioration of systemic status in SARS-CoV-2 infection is due to activation of the complement system [50].

The role of the viral spike protein of SARS-CoV-2 in the activation of an alternative complement pathway is perceptible. Both protein subunits S1 and S2 activate the alternative pathway of complement (APC) on the cell surface. A small molecule factor D inhibitor is proposed that actively blocks APC activation induced by SARS-CoV-2 spike proteins. APC is activated by the binding of the SARS$\mathrm{CoV}-2$ spike protein to heparan sulphate (HS) on the cell surface, which results in disruption of the function of factor $\mathrm{H}$. Studies suggest that the binding of the SARS-CoV-2 spike protein to HS leads to disruption of the correct conformation of factor $\mathrm{H}$ involved in the breakdown of the $\mathrm{C} 3$ convertase of the APC. Elevated levels of C5a and C5b-9 have been observed in moderate to severe patients. Studies using a C5 monoclonal antibody called ravulizumab have determined that it prevents the accumulation of C5b-9 in the body but does not prevent the activation of the complement system. Therefore, factor $\mathrm{H}$-targeted complement inhibitors may be more effective in the treatment of COVID-19 [50].

\section{Effect on viral replication}

Ion channel blockers. The SARS-CoV-2 viral ion channel is a complex of protein E, which in contrast to viral S protein, retains highly conserved sequences in its structure showing 93\% similarity in SARS-CoV and SARS-CoV-2. For the S protein, the similarity among coronaviruses is only $76.2 \%$. The conservativeness of protein E suggests that ion channels can be used in antiviral therapies. Protein E is an essential building block of the virus, and is involved in many aspects related to the life cycle of the virus: in the formation of the viral envelope, multiplication and release from host cells, and the maintenance of the microenvironment of the host cell. Inhibition of protein E may weaken or suppress the virulence of SARS-CoV-2 [51]. As part of the preliminary studies in the form of a search of the database of available agents that affect the action of ion channels, eight compounds were obtained from the 2,839 in the database. The ion channel blockers selected in the screening study were azacitidine, plerixafor, mebrofenin, mavorixafor, cyclen, kasugamycin and saroglitazar [51].

Ivermectin. An antiparasitic agent with a wide spectrum of action. The efficacy of ivermectin in vitro has been proved in reducing the RNA titre of viruses including the Dengue virus, West Nile virus and influenza virus. The IMPa/ $\beta 1$ importin transport protein heterodimer complex, which binds to SARS-CoV proteins in the cytoplasm, and then transported to the nucleus via the nuclear pore complex, is expected to play a significant role in the postulated mechanism of virus entry into host cells [52]. In the cell nucleus, the protein complex of the virus breaks down. Ivermectin binds and destabilises the IMPa/ $\beta 1$ complex, preventing the binding of the IMP $\alpha / \beta 1$ heterodimer to the viral protein. The described mechanism of action of ivermectin is likely to inhibit SARS$\mathrm{CoV}-2$ infection. In vitro studies by a team of researchers led by Caly [52] showed antiviral activity of ivermectin. A Vero/ human signalling lymphatic activation molecule (hSLAM) cell line infected with VIC01 SARS-CoV-2 isolated in Australia was used for the study and the efficacy of ivermectin was tested by RT-PCR assay. After 48 hours, there was a 5,000-fold reduction in viral RNA. Ivermectin manifests no toxic effects. It is a drug approved by the US Food and Drug Administration (FDA) for anti-parasitic therapy, and its antiviral activity against HIV-1 and Dengue, West Nile, Venezuelan equine encephalitis, and influenza viruses, has been verified. Such a broad spectrum of action is due to the dependence of many RNA viruses on the importin transport protein IMPa/ $\beta 1$, of which ivermectin is an inhibitor [52]. However, in order to impart a comparable effect in clinical conditions to that obtained in vitro, it would be necessary to achieve a much higher concentration of the drug in the body than is currently allowed when ivermectin is used for its antiparasitic effect, and potential side-effects may limit the originally expected positive effect. However, in a very small number of countries, ivermectin is, or has been, approved for use in COVID-19 [52].

\section{Multi-modal and synergistic action}

Brilacidin. A drug that has shown strong antiviral activity against SARS-CoV-2 in cell-line studies. Brilacidin is a small, synthetically produced molecule that is a non-peptide mimetic. It has antiviral properties both as monotherapy and in combination with remdesivir [53]. Studies conducted on Vero cell lines have shown the ability of brilacidin to reduce viral load, even in the early stages of infection, by interfering with the interaction of the SARS-CoV-2 spike protein with the host cell receptor ACE2. Further studies are planned to demonstrate the activity of brilacidin as an inhibitor of host cell viral infiltration and viral replication. An effect on replication of the main viral 3C-like protease has been demonstrated by analysis in silico. The aim of further research is to select drugs which affect many stages of the viral replication cycle [53].

Amantadine. A medicine is used to treat Parkinson's disease and is integral to the process of dopamine release. It was also used to treat H1N1 flu in 1976, but is not currently recommended for the treatment of infections with this virus. Studies on amantadine as a COVID-19 therapeutic showed that a group of patients diagnosed with the disease and administered the drug recovered, and in Parkinson's patients treated with amantadine, SARS-CoV-2 infection was asymptomatic [54]. In SARS-CoV-2 studies, amantadine has been shown to interfere with the multiplication of the virus in host cells. Ongoing in vitro investigations into the effectiveness of amantadine using Vero E6 cells lines have not shown any antiviral effect of this therapeutic agent. A number of scientific reports have suggested the possibility of 
amantadine inhibiting the binding of the virus spike protein to the human ACE2 receptor, but these theories have not been confirmed in laboratory studies. However, analyses of the mechanisms of action of SARS-CoV-2 indicate antiviral potential for amantadine. Literature reports indicate that by inhibiting viral enzymes, it inhibits viral entry into host cells and the release of RNA from the capsid. Another target for amantadine's action are viral ion channels: amantadine entering the channel formed by the SARS-CoV-2 E protein inhibits conduction in the channel $[51,54]$. SARS-CoV-2 causes damage to the central nervous system, which can lead to acute respiratory failure. Amantadine is a potent $\mathrm{N}$-methyl-D-aspartate glutamate receptor antagonist, which may suggest a protective effect in the treatment of COVID-19. The clinical effect on which the publications are based is only a description of individual cases and observations. Numerous clinical trials are currently under way to evaluate the efficacy of antiviral therapy with amantadine [55].

Chloroquine / hydroxychloroquine. The original drugs proposed to inhibit the process of virus entry into infected cells were chloroquine and hydroxychloroquine, antimalarial drugs that act at multiple stages of viral replication. They have analgesic, anti-inflammatory and immunomodulatory effects, inhibiting the production of cytokines. The main postulated specific mechanism of action of chloroquine and hydroxychloroquine was inhibition of terminal phosphorylation of ACE2 and elevation of the $\mathrm{pH}$ level in the endosomes, which would prevent the virus from entering the host cells by interfering with the glycosylation of the cell receptor. The drugs would then block intracellular transport. In addition, blocking MAP kinase would inhibit the process of virion folding in the final phase of viral replication [8]. Clinical trials in patients hospitalised for COVID-19 and treated with hydroxychloroquine in combination with azithromycin did not show therapeutic efficacy. There was no improvement in patients' health compared to the control group. The results of the trials led to the withdrawal of the initial recommendations for the use of these drugs [56].

Metal compounds. These are a group of medicines the properties of which may indicate activity potentially useful for the treatment of SARS-CoV-2 infections. Such drugs are based on individual metals from a wide range and their pharmacological properties are due to the electron structure of the metal, oxidation state, redox potential, etc. Their action can be directed to individual elements of pathogenesis or have a systemic effect. The mechanisms of metal-based drug action range broadly and they may affect cells by directly inhibiting enzymes, altering transcription factors, interacting with various biomolecules, increasing lipophilicity, altering cell membrane function, or disrupting the cell cycle. Among the proposed metal-based drugs is auranofin containing gold, which is currently used to treat rheumatoid arthritis, although its effect has not been fully understood. Tests carried out in vitro in Huh7 cell lines infected with SARSCoV-2 showed a $70 \%$ and $85 \%$ reduction in viral load in the supernatant after 24 hours and 48 hours, respectively, while intracellular RNA was reduced by $85 \%$ and $95 \%$ at the same intervals. In addition, auranofin has been shown to be able to reduce the expression of SARS-CoV-2-induced cytokines in Huh7 cell culture. Other COVID-19 treatment candidates are bismuth salts, the activity of which has been reported with respect to nucleotide triphosphatase, RNA helicase and the non-structural protein nsp13 [57].

Natural substances. Products of natural origin from traditional Chinese medicine can interact with receptors by inhibiting the penetration of the virus into the host cell, or inhibiting the process of viral replication. Inhibitors of proteins involved in viral replication proposed by Jin et al. [58] include the traditional Chinese preparations also known in contemporary Western medicine as ebselen, disulfiram, tideglusib, carmofur, shikonin, and imidazole derivative PX-12. Other triterpenes which interact with viral proteins and are commonly found in nature are oleanolic acid, ursolic acid, and hederagenin. In addition, mixtures of ascorbic acid, curcumin and glycyrrhizic acid have been shown to bind to viral proteins. Substances of natural origin exhibiting similar properties to antiviral drugs, i.e. nelfinavir and lopinavir, are kaempferol, quercetin, lutein, naringenin, and coumarin. Flavonoid derivatives of rosmarinic acid and the indole derivative amantadine are other examples of compounds of natural origin that have been shown to interact with the 3C-like viral protease. All the compounds of natural origin mentioned have similar mechanisms of action to antiviral drugs [7].

Echinacea. Due to their strong antiviral and immunomodulatory properties which affect macrophages and NK cells, extracts of Echinacea species plants have been considered for use in the treatment of COVID-19. Extracts obtained mainly from E. purpurea contain caftaric acid, chicory acid, caffeic acid, ferulic acid, chlorogenic acid, polysaccharides, and glycoproteins [59]. In in vitro studies on cell lines it was observed that the antiviral effect of Echinacea extracts is limited only to the phases before the virus enters the host cell. The putative mechanism of action of the extracts is based on the interaction with the structural proteins of the viral glycoprotein $S$ or the membrane protein $M$; therefore, the extracts may play a significant role in protection against infection as prophylactic agents [60].

\section{Preparations of indeterminate mechanism of action}

Imatinib. A tyrosine kinase inhibitor and a drug that has been on the market for over 20 years, which is used in anticancer therapies [61]. In treating COVID-19, the main goals for its administration were to reduce alveolar oedema and pulmonary capillary leakage. On the other hand, its antiviral properties have been confirmed in vitro, but subsequent studies have not confirmed its effect on replication or the process of virus penetration into host cells. Further studies on the antiviral mechanism of imatinib are required [62].

\section{CONCLUSIONS}

There are thousands of studies in the world aimed at finding preparations against the SARS-CoV-2 virus which may be useful in the treatment of COVID-19. The research directions discussed in this article indicate a high chance of effective therapeutics being obtained acting at different stages of SARS-CoV-2 infection in the near future. Having medicines at the clinician's disposal is particularly important in the context of the emergence of new strains of the virus, and the possible consequent ineffectiveness of vaccinations, and the 
presence in the population of people with immunological deficiencies and those who have not been vaccinated.

\section{Acknowledgments}

The authors would like to thank Prof. dr. hab. Stanisław Majcherczyk for his support in suggesting the appropriate scope of coverage of this manuscript.

\section{REFERENCES}

1. WHO Coronavirus (COVID-19) Dashboard. Who.int. Accessed August 1, 2021. https://covid19.who.int/

2. Hartenian E, Nandakumar D, Lari A, et al. The molecular virology of coronaviruses. J Biol Chem. 2020; 295(37): 12910-12934. doi: 10.1074/ jbc.REV120.013930

3. Rambaut A, Holmes EC, Toole ÁO, et al. A dynamic nomenclature proposal for SARS-CoV-2 lineages to assist genomic epidemiology. Nature Microbiol. 2021; 5(11): 1403-1407. doi: 10.1038/s41564-0200770-5.A

4. CDC. Science brief: Emerging SARS-CoV-2 variants. Cdc.gov. Published March 26, 2021. Accessed August 1, 2021. https://www. cdc.gov/coronavirus/2019-ncov/science/science-briefs/scientific-briefemerging-variants.html

5. Hacisuleyman E, Hale C, Saito Y, et al. Vaccine Breakthrough Infections with SARS-CoV-2 Variants. N Engl J Med. 2021; 384(23): 2212-2218. doi: 10.1056/nejmoa2105000

6. Alibek K, Tskhay A. Ahead of a vaccine: A safe method of protection against COVID-19 exists. Res Ideas Outcomes. 2020; 6. doi: 10.3897/ rio.6.e61709

7. Pawełczyk A, Zaprutko L. Anti-COVID drugs: Repurposing existing drugs or search for new complex entities, strategies and perspectives. Future Med Chem. 2020; 12(19): 1743-1757. doi: 10.4155/fmc-2020-0204

8. McKee DL, Sternberg A, Stange U, et al. Candidate drugs against SARS CoV-2 and COVID-19. Pharmacol Res. 2020; 157(104859): 104859.

9. Pawlik L, Śpiołek E, Fichna J, et al. Charakterystyka wirusa SARSCoV-2 i potencjalne farmakologiczne sposoby leczenia. Postępy Biochem. 2020; 66(2): 83-90. doi: 10.18388/pb.2020_321

10. Pereda R, González D, Rivero HB, et al. Therapeutic effectiveness of interferon- $\alpha 2 b$ against COVID-19: The cuban experience. J Interf Cytokine Res. 2020; 40(9): 438-442. doi: 10.1089/jir.2020.0124

11. Nakhlband A, Fakhari A, Azizi H. Interferon-beta offers promising avenues to COVID-19 treatment: a systematic review and meta-analysis of clinical trial studies. Naunyn Schmiedebergs Arch Pharmacol. 2021; 394(5): 829-838. doi: 10.1007/s00210-021-02061-x

12. Monk PD, Marsden RJ, Tear VJ, et al. Safety and efficacy of inhaled nebulised interferon beta-1a (SNG001) for treatment of SARS-CoV-2 infection: a randomised, double-blind, placebo-controlled, phase 2 trial. Lancet Respir Med. 2021; 9(2): 196-206.

13. Merzon E, Green I, Vinker S, et al. The use of aspirin for primary prevention of cardiovascular disease is associated with a lower likelihood of COVID-19 infection. FEBS J. 2021; (febs.15784). doi: $10.1111 /$ febs. 15784

14. Horby P, Lim WS, Emberson JR, et al. Dexamethasone in hospitalized patients with Covid-19 N Engl J Med. 2021; 384(8): 693-704.

15. Tardif J-C, Bouabdallaoui N, L'Allier PL, et al. Efficacy of colchicine in non-hospitalized patients with COVID-19. bioRxiv. Published online 2021: 2021.01.26.21250494. doi: 10.1101/2021.01.26.21250494

16. Manenti L, Maggiore U, Fiaccadori E, et al. Reduced mortality in COVID-19 patients treated with colchicine: Results from a retrospective, observational study. PLoS One. 2021; 16(3): e0248276

17. Allocetra-OTS in COVID-19 Phase II. Centerwatch.com. Accessed September 18, 2021. https://www.centerwatch.com/clinical-trials/ listings/255007/allocetra-ots-in-covid-19-phase-ii/?\&radius $=50 \& q u$ ery=Fever\&smt=true

18. BioSpace. Enlivex Reports Positive Top-Line Results from Phase II Clinical Trial Evaluating Allocetra in Severe and Critical COVID-19 Patients and Provides a Program Update. Biospace.com. Published February 3, 2021. Accessed September 18, 2021. https://www.biospace. com/article/releases/enlivex-reports-positive-top-line-results-fromphase-ii-clinical-trial-evaluating-allocetra-in-severe-and-criticalcovid-19-patients-and-provides-a-program-update/

19. Zhang X, Zhang Y, Qiao W, Zhang J, Qi Z. Baricitinib, a drug with potential effect to prevent SARS-COV-2 from entering target cells and control cytokine storm induced by COVID-19. Int Immunopharmacol. 2020; 86(106749): 106749.

20. The U.S. Food and Drug Administration. Information for Olumiant (baricitinib) tablets, for oral use. Access 25 April 2020. https://www. accessdata.fda.gov/drugsatfda_docs/label/2018/207924Orig1s000lbl.pdf

21. Yang G, Li Z, Mohammed I, et al. Identification of SARS-CoV-2-against aptamer with high neutralization activity by blocking the RBD domain of spike protein 1. Signal Transduct Target Ther. 2021; 6(1). doi: 10.1038/ s41392-021-00649-6

22. Sun M, Liu S, Wei X, et al. Aptamer Blocking Strategy Inhibits SARSCoV-2 Virus Infection. Angew Chemie - Int Ed. 2021; 60(18): 1026610272. doi: 10.1002/anie.202100225

23. Milewska A, Kaminski K, Ciejka J, Kosowicz K, Zeglen S, et al. HTCC: Broad Range Inhibitor of Coronavirus Entry. Plos One 2016; 11(6): e0156552. https://doi.org/10.1371/journal.pone.0156552

24. Uckun FM, Saund S, Windlass H, Trieu V. Repurposing anti-malaria phytomedicine Artemisinin as a COVID-19 drug. Front Pharmacol. 2021; 12: 649532

25. Horby PW, Pessoa-Amorim G, Peto L, et al. Tocilizumab in patients admitted to hospital with COVID-19 (RECOVERY): preliminary results of a randomised, controlled, open-label, platform trial. bioRxiv. 2021: 2021.02.11.21249258. doi: 10.1101/2021.02.11.21249258

26. Cohen MS. Monoclonal antibodies to disrupt progression of early covid-19 infection. N Engl J Med. 2021; 384(3): 289-291.

27. Marovich M, Mascola JR, Cohen MS. Monoclonal antibodies for prevention and treatment of COVID-19. JAMA. 2020; 324(2): 131-132.

28. Simonovich VA, Burgos Pratx LD, Scibona P, et al. A Randomized Trial of Convalescent Plasma in Covid-19 Severe Pneumonia. N Engl J Med. 2021; 384(7): 619-629. doi: 10.1056/nejmoa2031304

29. Salazar E, Christensen PA, Graviss EA, et al. Significantly decreased mortality in a large cohort of Coronavirus disease 2019 (COVID-19) patients transfused early with convalescent plasma containing hightiter anti-severe acute respiratory syndrome Coronavirus 2 (SARSCoV-2) spike protein IgG. Am J Pathol. 2021; 191(1): 90-107.

30. Tirumalaraju D. Celltrion's Covid-19 drug regdanvimab meets Phase III endpoints. Clinicaltrialsarena.com. Published June 15, 2021. Accessed August 1, 2021. https://www.clinicaltrialsarena.com/news/celltrionregdanvimab-phaseiii-data/

31. RenBio. RenBio completes \$24 million series A financing and licenses novel SARS-CoV-2 bispecific antibody from Columbia university. PR Newswire. Published May 20, 2021. Accessed August 1, 2021. https:// www.prnewswire.com/news-releases/renbio-completes-24-millionseries-a-financing-and-licenses-novel-sars-cov-2-bispecific-antibodyfrom-columbia-university-301295777.html

32. Gupta A, Gonzalez-Rojas Y, Juarez E, et al. Early Covid-19 Treatment With SARS-CoV-2 Neutralizing Antibody Sotrovimab. medRxiv. 2021: 2021.05.27.21257096. https://doi.org/10.1101/2021.05.27.21257096

33. Europa.eu. Accessed August 1, 2021. https://www.ema.europa.eu/en/ news/ema-reviewing-data-monoclonal-antibody-use-covid-19

34. A Phase 2 Trial of Infliximab in Coronavirus Disease 2019 (COVID-19). Clinicaltrials.gov. Accessed August 30, 2021. https://clinicaltrials.gov/ ct2/show/NCT04425538।

35. Ardestani A, Azizi Z. Targeting glucose metabolism for treatment of COVID-19. Signal Transduct Target Ther. 2021; 6(1): 1-2. doi: 10.1038/ s41392-021-00532-4

36. Larue RC, Xing E, Kenney AD, et al. Rationally designed ACE2-derived peptides inhibit SARS-CoV-2. Bioconjug Chem. 2021; 32(1): 215-223.

37. Xia S, Zhu Y, Liu M, et al. Fusion mechanism of 2019-nCoV and fusion inhibitors targeting HR1 domain in spike protein. Cell Mol Immunol. 2020; 17(7): 765-767. doi: 10.1038/s41423-020-0374-2

38. Zhao H, Zhou J, Zhang K, et al. A novel peptide with potent and broadspectrum antiviral activities against multiple respiratory viruses. Sci Rep. 2016; 6(February): 1-13. doi: 10.1038/srep22008

39. Ramakrishnan S, Nicolau DV Jr, Langford B, et al. Inhaled budesonide in the treatment of early COVID-19 (STOIC): a phase 2, open-label, randomised controlled trial. Lancet Respir Med. 2021; 9(7): 763-772.

40. Hoffmann M, Hofmann-Winkler H, Smith JC, et al. Camostat mesylate inhibits SARS-CoV-2 activation by TMPRSS2-related proteases and its metabolite GBPA exerts antiviral activity. EBioMedicine. 2021; 65(Ivm). doi: 10.1016/j.ebiom.2021.103255

41. Nojomi M, Yassin Z, Keyvani H, et al. Effect of Arbidol (Umifenovir) on COVID-19: a randomized controlled trial. BMC Infect Dis. 2020; 20(1): 1-10. doi: 10.1186/s12879-020-05698-w

42. Horby PW, Mafham M, Bell JL, et al. Lopinavir-ritonavir in patients admitted to hospital with COVID-19 (RECOVERY): a randomised, controlled, open-label, platform trial. Lancet. 2020; 396(10259): 13451352. doi: 10.1016/S0140-6736(20)32013-4 
43. Ahmad B, Batool M, Ain QU, Kim MS, Choi S. Exploring the binding mechanism of PF-07321332 SARS-CoV-2 protease inhibitor through molecular dynamics and binding free energy simulations. Int J Mol Sci. 2021; 22(17): 9124

44. Sheahan TP, Sims AC, Graham RL, et al. Broad-spectrum antiviral GS-5734 inhibits both epidemic and zoonotic coronaviruses. Sci Transl Med. 2017; 9(396). doi: 10.1126/scitranslmed.aal3653

45. Martin U. The Biologics News and Reports Portal. Pipelinereview. com. Accessed August 1, 2021. https://pipelinereview.com/ index.php/2021070178593/Small-Molecules/Ateas-AT-527-anOral-Antiviral-Drug-Candidate-Reduces-Viral-Replication-inHospitalized-Patients-with-COVID-19-in-Phase-2-Interim-Analysis. html

46. Good SS, Westover J, Jung KH, et al. AT-527, a double prodrug of a guanosine nucleotide analog, is a potent inhibitor of SARS-CoV-2 in vitro and a promising oral antiviral for treatment of covid-19. Antimicrob Agents Chemother. 2021; 65(4): 1-12. doi: 10.1128/ AAC.02479-20

47. Agrawal U, Raju R, Udwadia ZF. Favipiravir: A new and emerging antiviral option in COVID-19. Med J Armed Forces India. 2020; 76(4): 370-376. doi: 10.1016/j.mjafi.2020.08.004

48. Cox RM, Wolf JD, Plemper RK. Therapeutically administered ribonucleoside analogue MK-4482/EIDD-2801 blocks SARS-CoV-2 transmission in ferrets. Nat Microbiol. 2021; 6(1): 11-18. doi: 10.1038/ s41564-020-00835-2

49. Fischer W, Eron Jr JJ, Holman W, et al. Molnupiravir, an Oral Antiviral Treatment for COVID-19. medRxiv Prepr. 2021: 1-30. https://doi. org/10.1101/2021.06.17.21258639

50. Yu J, Yuan X, Chen H, et al. Direct activation of the alternative complement pathway by SARS-CoV-2 spike proteins is blocked by factor D inhibition. Blood. 2020; 136(18): 2080-2089.

51. Tomar PPS, Krugliak M, Arkin IT. Identification of SARS-CoV-2 E channel blockers from a repurposed drug library. Pharmaceuticals (Basel). 2021; 14(7): 604

52. Caly L, Druce JD, Catton MG, et al. The FDA-approved drug ivermectin inhibits the replication of SARS-CoV-2 in vitro. Antiviral Res. 2020; 178(104787): 104787.
53. Bakovic A, Risner K, Bhalla N, et al. Brilacidin, a COVID-19 Drug Candidate, Exhibits Potent In Vitro Antiviral Activity Against SARS-CoV-2. bioRxiv. 2020: 2020.10.29.352450. https://doi. org/10.1101/2020.10.29.352450

54. Aranda-Abreu GE, Aranda-Martínez JD, Araújo R, et al. Observational study of people infected with SARS-Cov-2, treated with amantadine. Pharmacol Reports. 2020; 72(6): 1538-1541. doi: 10.1007/s43440-02000168-1

55. Butterworth RF. Potential for the Repurposing of Adamantane Antivirals for COVID-19. Drugs R D. 2021; (0123456789). doi: 10.1007/ s40268-021-00351-6

56. Cavalcanti AB, Zampieri FG, Rosa RG, et al. Hydroxychloroquine with or without azithromycin in mild-to-moderate Covid-19. N Engl J Med. 2020; 383(21): 2041-2052.

57. Cirri D, Pratesi A, Marzo T, et al. Metallo therapeutics for COVID-19. Exploiting metal-based compounds for the discovery of new antiviral drugs. Expert Opin Drug Discov. 2021; 16(1): 39-46. doi: 10.1080/17460441.2020.1819236

58. Jin Z, Du X, Xu Y, et al. Structure of Mpro from SARS-CoV-2 and discovery of its inhibitors. Nature. 2020; 582(7811): 289-293.

59. Brendler T, Al-Harrasi A, Bauer R, et al. Botanical drugs and supplements affecting the immune response in the time of COVID-19: Implications for research and clinical practice. Phytother Res. 2021; 35(6): 3013-3031

60. Signer J, Jonsdottir HR, Albrich WC, et al. In vitro virucidal activity of Echinaforce ${ }^{\infty}$, an Echinacea purpurea preparation, against coronaviruses, including common cold coronavirus 229E and SARSCoV-2. Virol J. 2020; 17(1): 136

61. Bernal-Bello D, Morales-Ortega A, Isabel Farfán-Sedano A, de Tena JG, Martín-López JVS. Imatinib in COVID-19: hope and caution. Lancet Respir Med. doi: 10.1016/S2213-2600(21)00266-6

62. Aman J, Duijvelaar E, Botros L, et al. Imatinib in patients with severe COVID-19: a randomised, double-blind, placebo-controlled, clinical trial. Lancet Respir Med. 2021. doi: 10.1016/S2213-2600(21)00237-X 